\title{
Religiosität und Integration: drei Bemerkungen zu einer ambivalenten Beziehung
}

\author{
Heinz Faßmann
}

European societies have become more and more multiethnic and multireligious due to the large amount of immigration in recent decades. The core of the contribution deals with the role religious affiliation plays in the integration process. In theory, religion is an important unifying factor for newly arrived migrants, but this is of decreasing importance parallel to the increasing length of stay in the target country. In empirical studies, this time dependent process is hard to prove; it seems that other factors (qualification, country of origin) are more important. In specific circumstances, however, religion has become an important factor when migrants rely on specific traditions and norms, which are in conflict with that of the majority. These norm conflicts are presented exemplified by some court cases. The contribution ends with the recommendation that an overemphasizing religious influence should be reduced to make the integration process easier for the immigrant population.

Heinz Faßmann, born 1955 in Düsseldorf, studied Geography and History in Vienna, completed a postgraduate study (Sociology) at the Institute for Advanced Studies and earned his doctorate in 1980. He finished habilitation studies in 1992 and became C4professor of Geography and Geoinformatics at the TU Munich. He has been professor of Applied Geography, Spatial Research and Spatial Planning at the University of Vienna since 2000. He is Member of Academia Europaea and a full member of the Austrian Academy of Sciences. Moreover, he is Chairman of the Expert Council for Integration at the Federal Ministry of the Interior and member of the Expert Council of German Foundations on Integration and Migration. His research topics are: urban geography, demography (especially in the field of migration), spatial planning in Austria. He is author and editor of numerous scientific works.

\section{Vorbemerkung}

Der vorliegende Beitrag befasst sich mit Religion, Religiosität und Integration in einer pluralen Einwanderungsgesellschaft. Er ist im Wesentlichen dreigeteilt: Als erstes werden die quantitativen Veränderungen der Größe der Religionsgemeinschaften am Beispiel Österreichs gezeigt und auf die Bedeutung der Zuwanderung hingewiesen. Als zweites wird konzeptionell und empirisch der Zusammenhang von Religion und Religiosität auf der einen und Integration auf der anderen Seite dargestellt. Und schließlich werden ausgewählte und mehr oder minder religiös motivierte Konfliktsituationen aus der Arbeitswelt präsentiert, 
die zeigen, dass ein theoretisch erwarteter Anpassungsprozess nicht so friktionsfrei abläuft wie erwartet. Mit einem knappen Fazit endet der Beitrag.

\section{Signifikante Verschiebungen der religiösen Struktur}

Österreich, Deutschland und viele andere, wenn auch nicht alle Staaten Europas, sind zu Einwanderungsländern geworden - und das ohne politische Absicht und ohne Plan, vielmehr aufgrund der faktischen Ereignisse. Seit Beginn der 1960erJahre war in Österreich der internationale Wanderungssaldo fast immer positiv, in Deutschland bereits seit Mitte der 1950er-Jahre. Allein im vergangenen Jahrzehnt summierte sich in Österreich der kumulierte positive Wanderungssaldo auf mehr als 500.000 Personen. Ein mittelgroßes Bundesland kam einwohnermäßig in einer Dekade gleichsam hinzu.

Mit der Zuwanderung kam es auch zu einer quantitativen Verschiebung im Bereich der Religionsgemeinschaften und zu einer qualitativen Veränderung der Religiosität in der Gesellschaft. Die Säkularisierungsthese, die von einem generellen Bedeutungsverlust der Religionen - zumindest im öffentlichen Raum ausgeht, kann nicht mehr als uneingeschränkt und empirisch bewiesen angesehen werden (Lehmann 2004). Ganz im Gegenteil: Mit der Zuwanderung von Arbeitskräften, Flüchtlingen und Familienangehörigen aus Südosteuropa und dem Nahen Osten verzeichnete der Islam einen quantitativen Bedeutungsgewinn und stellt damit eine Religion dar, die zumindest für einen Teil der Gläubigen Mitgestalterin des täglichen Lebens ist. Ob das für die Integration förderlich ist oder ob dadurch nicht neue Normenkonflikte in der Gesellschaft auftreten, kann nicht ohne weiteres verneint oder bejaht werden.

Was sich mit Sicherheit feststellen lässt, ist die quantitative Veränderung der demographischen Struktur der Religionsgemeinschaften. Aus einem - vom Selbstverständnis und den Quantitäten her betrachtet - katholischen Land, in dem sich der Protestantismus und das Judentum erst nach und nach, und oft sehr konfliktreich, einen gesellschaftlichen Platz verschaffen konnten, wurde sehr rasch eine multireligiöse Gesellschaft.

Eine exakte und verlässliche statistische Abbildung dieser Veränderung ist aber nicht mehr möglich. In den Volkszählungen wurde immer nur nach dem Religionsbekenntnis gefragt - und dies auch nur bis 2001. Die Angabe des Religionsbekenntnisses ist zwar auf dem Meldezettel möglich, eine Überprüfung anhand von Dokumenten ist aber nicht vorgeschrieben. Seit 2001 können daher keine präzisen Angaben mehr über die Größe der Religionsgemeinschaften gemacht werden. Lediglich Selbstangaben über die Zahl der Personen, die den Kirchenbeitrag entrichten, sowie Schätzungen anhand der durchschnittlichen Ein- und Austritte sind möglich. Einschränkend muss auch gesagt werden, dass das Religionsbekenntnis selbst wenig über die tatsächliche Religiosität in der 
Bevölkerung aussagt, die Angaben über das Bekenntnis sind bestenfalls ein Hinweis. ${ }^{1}$

Was sich anhand der Volkszählungsergebnisse für Österreich dennoch zeigen lässt, ist der Rückgang der Bevölkerung, die sich zur römisch-katholischen Kirche bekennt, die in Summe aber noch immer die stärkste religiöse Kraft in Österreich darstellt. In den ersten beiden Volkszählungen nach dem Zweiten Weltkrieg gaben noch fast 90 \% der Bevölkerung an, der römisch-katholischen Kirche anzugehören, 2001 waren es nur mehr rund 74 \%. Die quantitativ zweitwichtigste Gruppe ist jene ohne Bekenntnis oder ohne Angaben. 1951 lag der entsprechende Wert noch bei rund $4 \%, 2001$ bereits bei rund $15 \%$. Einen quantitativen Rückgang verzeichnen auch die evangelischen Kirchen AB und HB: von rund $6 \%$ im Jahr 1951 auf knapp 5 \% im Jahr 2001, während die sich zum Islam bekennende Bevölkerung von statistisch nicht existent auf $4 \%$ im Jahr 2001 zunahm. Schließlich ist die Steigerung der Gruppe der „sonstigen Bekenntnisse“ zu erwähnen, die den Buddhismus ebenso mit einschließt wie die Zeugen Jehovas und 2001 bei $3 \%$ lag.

Tabelle 1: Wohnbevölkerung nach Religionsbekenntnis 1951-2001 (absolut und relativ)

\begin{tabular}{|l|r|r|r|r|r|r|}
\hline & $\mathbf{1 9 5 1}$ & $\mathbf{1 9 6 1}$ & $\mathbf{1 9 7 1}$ & $\mathbf{1 9 8 1}$ & $\mathbf{1 9 9 1}$ & $\mathbf{2 0 0 1}$ \\
\hline Röm.-katholisch & 6.170 .084 & 6.295 .075 & 6.548 .316 & 6.372 .645 & 6.081 .454 & 5.915 .421 \\
\hline Evangelisch & 429.493 & 438.663 & 447.070 & 423.162 & 388.709 & 376.150 \\
\hline Altkatholisch & 32.919 & 29.652 & 26.992 & 25.547 & 18.930 & 14.621 \\
\hline Israelitisch & 11.224 & 9.049 & 8.461 & 7.123 & 7.268 & 8.140 \\
\hline Islamisch & & & 22.267 & 76.939 & 158.776 & 338.988 \\
\hline Sonstiges & 23.093 & 31.386 & 74.440 & 118.866 & 197.433 & 255.681 \\
\hline Ohne Bekenntnis & 264.014 & 266.009 & 321.218 & 452.039 & 672.251 & 963.263 \\
\hline Ohne Angabe & 3.078 & 3.973 & 42.762 & 79.017 & 270.965 & 160.662 \\
\hline Insgesamt & 6.933 .905 & 7.073 .807 & 7.491 .526 & 7.555 .338 & 7.795 .786 & 8.032 .926 \\
\hline
\end{tabular}

1 Die Volkszählungsergebnisse von 2001 stellen die Grundlage für weitere Schätzungen dar. Statistik Austria hat anhand der Herkunftsangaben der Zuwanderer seit 2001 und einer dabei unterstellten Religionszugehörigkeit, die der Gesamtbevölkerung im Herkunftsland entspricht, die Werte extrapoliert. Das Projekt WIREL an der ÖAW baut seine Schätzung auf der Geburten- und Sterbeentwicklung nach Religionsbekenntnis und den geschätzten $\mathrm{Zu}$ - und Abwanderungen auf. Die ebenfalls zur Anwendung kommenden $\mathrm{Zu}$ - und Austritte aus den Religionsgemeinschaften können mangels statistischer Grundlagen nur für einzelne Religionsgemeinschaften durchgeführt werden, was die Ergebnisse verfälscht und nicht vergleichbar macht. 
Tabelle 1 (Fortsetzung)

\begin{tabular}{|l|r|r|r|r|r|r|}
\hline & $\mathbf{1 9 5 1}$ & $\mathbf{1 9 6 1}$ & $\mathbf{1 9 7 1}$ & $\mathbf{1 9 8 1}$ & $\mathbf{1 9 9 1}$ & $\mathbf{2 0 0 1}$ \\
\hline Röm.-katholisch & 89,0 & 89,0 & 87,4 & 84,3 & 78,0 & 73,6 \\
\hline Evangelisch & 6,2 & 6,2 & 6,0 & 5,6 & 5,0 & 4,7 \\
\hline Altkatholisch & 0,5 & 0,4 & 0,4 & 0,3 & 0,2 & 0,2 \\
\hline Israelitisch & 0,2 & 0,1 & 0,1 & 0,1 & 0,1 & 0,1 \\
\hline Islamisch & 0,0 & 0,0 & 0,3 & 1,0 & 2,0 & 4,2 \\
\hline Sonstiges & 0,3 & 0,4 & 1,0 & 1,6 & 2,5 & 3,2 \\
\hline Ohne Bekenntnis & 3,8 & 3,8 & 4,3 & 6,0 & 8,6 & 12,0 \\
\hline Ohne Angabe & 0,0 & 0,1 & 0,6 & 1,0 & 3,5 & 2,0 \\
\hline Insgesamt & 100,0 & 100,0 & 100,0 & 100,0 & 100,0 & 100,0 \\
\hline
\end{tabular}

Quelle: Statistik Austria

Aufgrund der Zuwanderung aus dem Ausland und einer Fortschreibung des bisherigen Ein- und Austrittsverhaltens der Bevölkerung kann mit Zunahmen bei den Gruppen „ohne Bekenntnis“, den Muslimen und den „,sonstigen Bekenntnissen“ gerechnet werden, wobei diese Veränderungen nur mehr auf Schätzungen basieren. Abnahmen müssen wohl die beiden christlichen Kirchen, die Katholiken und die Protestanten, zur Kenntnis nehmen, wobei bei den Protestanten der Effekt der Zuwanderung aus Deutschland möglicherweise eine Rolle spielt und eine weitere Abnahme verhindert.

Migration stellt jedenfalls eine wichtige Triebfeder der quantitativen Veränderungen der Religionsgemeinschaften dar, aber nicht die alleinige. Die Abnahme der christlichen Bekenntnisse kann nicht auf Abwanderung der Mitglieder der entsprechenden Religionsgemeinschaften zurückgeführt werden, denn diese erfasst die „eingesessenen“ Religionsgemeinschaften nur marginal, sondern auf eine Abkehr von der Kirche und möglicherweise auch vom Glauben der im Land wohnhaften Bevölkerung - Stichwort Säkularisierung. Umgekehrt ist klar, dass die Zunahme des Islam im Wesentlichen auf die Zuwanderung aus Drittstaaten zurückzuführen ist, ebenso die Zunahme der „sonstigen Bekenntnisse“. Die wachsende Diversität der Herkunftsgebiete, aus denen sich die internationale Zuwanderung nach Österreich speist, hat die religiöse Pluralisierung jedenfalls maßgeblich mitbestimmt. Gleiches gilt für Deutschland, die Schweiz, Frankreich und viele andere Staaten Europas. 


\section{Religion und Integration: eine ambivalente Beziehung}

Dieser empirische Befund führt zur zweiten Frage dieses Beitrags: Ist wachsende religiöse Pluralität hinderlich für einen raschen Integrationsprozess, oder verhält sich religiöse Pluralität integrationspolitisch neutral? Um diese Frage zu beantworten, ist eine knappe Definition des Integrationsbegriffes notwendig. Der Autor folgt dabei Bommes (2004), der unter Integration die messbare und auch politisch zu fördernde Teilhabe von Zugewanderten oder schon im Inland Geborenen an den zentralen Bereichen des gesellschaftlichen Lebens versteht (vgl. Bommes 2004). Die zentralen Bereiche des gesellschaftlichen Lebens umfassen dabei das Bildungssystem, den Arbeitsmarkt und das politische System. Integration bedeutet aufgrund dieses Begriffsverständnisses Teilhabe am gesellschaftlichen Leben, Aufnahme und Mitarbeit in den wichtigen gesellschaftlichen Institutionen sowie Eingliederung ,in ein größeres Ganzes“. Religion kann bei diesem Prozess der Teilhabe an und der Eingliederung in die Gesellschaft Hemmnis und Hilfe zugleich sein (Langenfeld 2010).

\subsection{Der Race Relation Cycle und die Zugehörigkeit zu religiösen Gemeinschaften}

Die Chicagoer Schule der Soziologie hat in ihrem Konzept des Race Relation Cycles nicht der Religion per se, dafür aber den religiösen und ethnischen Gemeinschaften eine besondere Bedeutung für den Integrationsprozess eingeräumt. ${ }^{2}$ Der Race Relation Cycle wird an dieser Stelle erwähnt, weil er als Referenzmodell eines linearen und friktionsfrei ablaufenden Integrationsprozesses lange Zeit die politische Debatte beherrschte. Dass die wissenschaftliche Forschung weder mit dem normativen Charakter dieses Konzepts einverstanden war, noch die allgemeine Gültigkeit empirisch nachweisen konnte, wird an dieser Stelle angeführt, aber nicht im Detail erläutert (vgl. Treibel-Illian 2011).

Der Race Relation Cycle stellt eine Verallgemeinerung der beobachteten Integrationsprozesse der vor und nach dem Ersten Weltkrieg nach Chicago zugewanderten Bevölkerung dar (vgl. Park / Burgess 1921). Er gliedert sich in vier Phasen - Aufnahme, Wettbewerb, Akkulturation und Assimilation -, die gleichsam automatisch ablaufen.

In der ersten Phase kommt es - so das idealtypische Modell - zur Aufnahme von Zuwanderern durch die Angehörigen ihrer jeweiligen ethnischen und religiösen Bezugsgruppe. Diese Gemeinschaften gewähren Unterkunft, Einbettung in ein soziales Gefüge und sozioökonomische Absicherung. Die Erstintegration in

2 Der englische Begriff ,race“ ist bekanntlich nicht mit dem deutschen Begriff „Rasse“ zu übersetzen, sondern vielmehr mit „ethnischer Zugehörigkeit“. 
den Aufnahmekontext wird durch die entsprechende ethnische und religiöse Gemeinschaft gewährleistet.

In einer zweiten Phase - abermals die Modellannahme - treten Zuwanderer aus dem Kontext ihrer ethnischen und religiösen Gemeinschaften (und aus den entsprechenden Wohnvierteln) heraus und stellen sich der Konkurrenz auf dem Arbeits- und Wohnungsmarkt. Im Zuge dieses durch den Markt vermittelten Prozesses wird ersichtlich, in welchen Bereichen welches Ausmaß an Akkulturation notwendig ist: Das Erlernen der Sprache der Mehrheitsgesellschaft wird notwendig, um mit dieser zu kommunizieren; berufliche Qualifikationen müssen ergänzt oder erneuert werden, um in einem Wettbewerb gegenüber Konkurrenten bestehen zu können; aber auch ein sozial normiertes Verhalten ist notwendig, um einen Miet- oder Arbeitsvertrag zu erhalten.

Die Konkurrenzsituation leitet die Akkulturierungsphase ein und damit das Hineinwachsen der Zugewanderten in die neue kulturelle Umwelt. Die Bindungskraft zur jeweiligen ethnischen und religiösen Gemeinschaft, die sich immer auch in den entsprechenden Nachbarschaften manifestiert, nimmt mit fortschreitender Aufenthaltsdauer ab. Die Zugewanderten lösen sich davon und wechseln auch den Wohnstandort, um in eine neue kulturelle Umwelt integriert zu werden.

Am Ende der Akkulturierung und des gesamten Race Relation Cycle steht schließlich die Assimilation. Assimilation bedeutet dabei vollständige Anpassung der Zugewanderten an die Werte, Normen und kulturellen Gepflogenheiten der Mehrheitsgesellschaft. „Assimilation is a process of interpenetration and fusion in which persons and groups acquire the memories, sentiments, and attitudes of other persons or groups, and, by sharing their experience and history, are incorporated with them in a common cultural life." (Park / Burgess 1921)

Der Race Relation Cycle schreibt also der Zugehörigkeit zu ethnischen und religiösen Gemeinschaften zunächst positive Effekte zu. Sie sorgt am Beginn des Prozesses für soziale Einbettung und psychische Stabilität. Mit ansteigender Aufenthaltsdauer sollten die Bindungskräfte aber abnehmen. Wenn dies nicht der Fall ist, dann hat dies eher einen negativen Effekt. Ethnische und religiöse Gemeinschaften segregieren sich von der Aufnahmegesellschaft, bilden möglicherweise eine dauerhafte „Parallelgesellschaft“, die für eine Minimierung der AuBenkontakte sorgt und eine eigene Welt in der Aufnahmegesellschaft entstehen lässt. Die Zugehörigkeit zu ethnischen und religiösen Gemeinschaften wirkt dann auch negativ auf den Integrationsprozess ein, wenn sie eine „in-group“-Orientierung fördert und damit die Interaktion mit der Aufnahmegesellschaft behindert. 


\subsection{Empirische Befunde zum Einfluss von Religion und Religiosität}

Soweit zur konzeptionellen Vorgabe: Empirisch lassen sich positive und negative Effekte religiöser Gemeinschaften auf den Integrationsprozess abbilden, auch wenn die über Einzelfallstudien hinausgehende Forschungslage eher dürftig ist. Untersucht wurde meistens der Einfluss der Zugehörigkeit zu einer Religionsgemeinschaft auf Bildung und Schulbesuch. Keine aktuellen Studien sind zu den anderen Integrationsdimensionen bekannt, insbesondere auch nicht mit der Koppelung an die Aufenthaltsdauer, die bei der Chicagoer Schule einen wichtigen Stellenwert einnimmt. In welchem Ausmaß beispielsweise die Religiosität die Erwerbstätigkeit in Abhängigkeit zur Aufenthaltsdauer steuert, so wie es Max Weber in seiner klassischen Arbeit über die protestantische Ethik und den Kapitalismus am Beginn des 20. Jahrhunderts untersucht hat, kann zumindest für Österreich und Deutschland aktuell nicht ausgesagt werden (Winckelmann 1984).

Die wenigen ernsthaften und ernstzunehmenden empirischen Studien über den Einfluss der Religiosität auf den Integrationsprozess beziehen sich auf die USA und zeigen einen positiven Zusammenhang zwischen Bildungsaspiration und Bildungserfolgen auf der einen Seite und Religiosität und Zugehörigkeit zu religiösen Gemeinschaften auf der anderen Seite. Vor allem jüdische, buddhistische und muslimische Schüler in den USA erreichen durchschnittlich höhere Bildungsabschlüsse als Personen anderer Religionszugehörigkeiten (vgl. Sander 2010; Zhou / Bankston 1998). Eine verstärkte soziale Kontrolle durch die ethnisch-religiöse Gemeinschaft sowie die zusätzlichen Bildungsangebote (Hausaufgabenhilfe, Sprach- und Nachhilfekurse), die von der Gemeinschaft bereitgestellt werden, sind dafür wohl verantwortlich, und nicht der Inhalt der religiösen Glaubenslehre.

In Deutschland ist dieser „positive Diasporaeffekt“ nur für Protestanten bzw. Katholiken nachweisbar, wenn die jeweilige Konfession sich in einer Minderheitensituation befindet (Helbig / Schneider 2014), also etwa bei Katholiken, die in einer mehrheitlich protestantischen Region leben, und umgekehrt. Bei Muslimen in Deutschland oder Österreich ist so ein positiver Diasporaeffekt, der zu einer verstärkten Anstrengungsbereitschaft führt, nicht feststellbar - ganz im Gegenteil. Dies kann besonders bei Frauen beobachtet werden: Je stärker die Religiosität, desto häufiger spielt sich das Leben in der Diaspora ab und desto seltener wird eine außerhäusliche Erwerbsarbeit gesucht. Stichs und Müssig zeigen auf Basis einer empirischen Erhebung zum Thema „Muslimisches Leben in Deutschland“, dass muslimische Frauen bei sonst gleichen Voraussetzungen wie Bildung, Alter und familiärer Situation signifikant seltener erwerbstätig sind. Darüber hinaus analysierten die Autorinnen den Zusammenhang zwischen Religiosität und einem darauf basierenden traditionellen Lebensstil. Das Tragen eines Kopftuchs - ein Umstand, der leicht abfragbar ist - diente dabei als Näherungsvariable. Es zeigte sich, dass kopftuchtragende Muslimas bei gleicher Bil- 
dung, gleichem Alter und gleicher familiärer Situation seltener erwerbstätig sind als nicht-kopftuchtragende Muslimas (Haug / Stichs / Müssig 2009).

Religiös konnotierte Gemeinschaften können in solchen Situationen zum Integrationshemmnis werden. Sie geben ihre Bindung nicht auf und halten ihre Angehörigen davon ab, die zweite und dritte Phase des Race Relation Cycles zu beginnen. Solche religiös konnotierten Gemeinschaften prägen kulturelle, moralische und vielleicht sogar rechtliche Normaussagen, die im Widerspruch zu Normaussagen des Staates oder der Mehrheitsgesellschaft stehen können. Insbesondere dann, wenn der religiöse Eifer keinen Spielraum für Annäherung und Kompromiss frei lässt oder wenn die sozialen Normaussagen sich nicht nur auf die grundsätzlichen Fragen beschränken, sondern auch das tägliche Leben bestimmen. Religion wird dann zu einem Integrationshemmer, wenn sie kompromisslos versucht, Menschen das Eintauchen in das Normen- und Wertegerüst der Mehrheitsgesellschaft moralisch zu verwehren. Der positive Effekt durch Gemeinschaftsbildung wird durch den negativen Effekt der Distanzwahrung konterkariert, Religion wird zu einem Ambivalenzphänomen: Sie trägt die Chance zum gedeihlichen Zusammenleben ebenso in sich wie die Potenz zum Konflikt.

\section{Akkulturierung: Brüche und Konflikte}

Brüche und Konflikte im Rahmen der Akkulturierung von Zugewanderten ergeben sich bei jenen religiösen Gemeinschaften, deren Werte und Normen in einem Gegensatz zu den gängigen Werten und Normen der Mehrheitsgesellschaft stehen. Diskutiert wird dieser Gegensatz derzeit besonders häufig im Zusammenhang mit dem Islam, weil sich dessen Regelungsanspruch auf viele Lebensbereiche ausdehnt. Nicht alle Formen religiöser Wertvorstellungen lassen sich in gleicher Weise mit dem freiheitlichen Ethos der Moderne vereinbaren. Anhand von vier ausgewählten Fällen aus der Schul- und Arbeitswelt werden solche Normkollisionen exemplifiziert. Auf andere Konfliktsituationen, die unterschiedliche Rechtsgüter betreffen - z. B.: Religionsfreiheit versus das Recht auf körperliche Unversehrtheit (Beschneidung von Knaben) -, wird an dieser Stelle aufmerksam gemacht, sie werden aber nicht weiter erläutert (SVR 2016). ${ }^{3}$

Die folgenden vier Beispiele wurden dem Jahresgutachten des SVR 2016 entnommen und werden verkürzt dargestellt. Sie beziehen sich auf Konflikte zwischen privaten Arbeitgebern und Arbeitnehmern und greifen damit etwas auf, das im Race Relation Cycle als automatisch ablaufend eingeschätzt wurde, denn

3 Das Jahresgutachten 2016 des Sachverständigenrates deutscher Stiftungen für Integration und Migration hat diese Normenkonflikte zum zentralen Inhalt. Die angeführten Beispiele wurden dem Jahresgutachten 2016 entnommen. Dem SVR gehören neun Wissenschaftlerinnen und Wissenschaftler aus verschiedenen Disziplinen und Forschungsrichtungen an (nähere Informationen, siehe Internetquellen: SVR). 
die Bedingungen der Erwerbsarbeit oder der schulischen Umwelt führen offensichtlich nicht automatisch zur Anpassung und in weiterer Folge zur Assimilation. Ganz im Gegenteil: Der Akkulturierungsprozess bleibt gleichsam stecken und wird zur Klärung an die Gerichte weitergereicht.

Der erste Fall: Eine Verkäuferin muslimischen Glaubens war seit ihrer Ausbildung in einem Kaufhaus im ländlichen Raum insgesamt fast 10 Jahre tätig, bevor sie aus religiösen Gründen beschloss, ein Kopftuch zu tragen. Sie teilte dies der Personalabteilung vor ihrer Rückkehr aus einem Erziehungsurlaub mit und begründete es damit, dass sich ihre religiösen Vorstellungen gewandelt hätten und ihr Glaube es verbiete, sich in der Öffentlichkeit ohne Kopftuch zu zeigen. Das Kaufhaus räumte der Frau zunächst Bedenkzeit ein und kündigte den Vertrag, nachdem die Verkäuferin auf ihrer Entscheidung beharrte. Das Landesarbeitsgericht Hessen gab dem Arbeitgeber zunächst recht, das Bundesarbeitsgericht stellte jedoch fest, der Arbeitgeber könne zwar aufgrund seines Direktionsrechts durchaus betriebliche Bekleidungsregeln festlegen, diese müssten aber die grundrechtlich geschützte Glaubensfreiheit berücksichtigen. Dieses Recht wiege schwerer als die Sorge des Arbeitgebers, wegen ausbleibender Kunden Umsatzeinbußen zu erleiden, und sei somit stärker zu berücksichtigen als dessen grundrechtlich geschützte unternehmerische Freiheit (BAG, Urt. v. 10 Oktober 2002, Az. 71/02; SVR 2016).

Der zweite Fall handelt von einem muslimischen Lagerarbeiter, der sich im Zuge der Tätigkeit in einem Supermarkt geweigert hatte, alkoholische Getränke in die Verkaufsregale einzuordnen, und daraufhin gekündigt wurde. Der Fall kam nach einem erstinstanzlichen Urteil zum Bundesarbeitsgericht, welches seinerseits die Causa an das zuständige Landesarbeitsgericht zur Klärung der Frage, ob der Arbeitnehmer bereits bei Vertragsabschluss vom gegenwärtigen Aufgabenprofil wusste, zurückverwies. Wäre dies der Fall gewesen, dann wäre er auch zu Arbeiten verpflichtet gewesen, die seiner Religion aus subjektiver Sichtweise zuwiderlaufen könnten. Wenn er bei Vertragsabschluss nicht damit rechnen musste, auch nur mittelbar am Verkauf alkoholischer Getränke beteiligt zu sein, dann möge der Arbeitgeber ihm einen Ersatzarbeitsplatz zuweisen (BAG, Urt. v. 24. Februar 2011, Az. 2 AZR 636/09; SVR 2016). Im konkreten Fall haben sich die Konfliktparteien verglichen. Dennoch zeigt auch dieser Fall eine gewisse Form von Absurdität sowie einen Missbrauch religiöser Inhalte, denn die wirklichen Ursachen der Konflikte liegen wohl eher in einer Unzufriedenheit des Arbeitnehmers mit der Arbeitssituation bzw. des Arbeitgebers mit der Arbeitsleistung des Arbeitnehmers.

In der bekannten „Kopftuch-Frage“ bei einem öffentlich-rechtlichen Dienstverhältnis ist die Abwägung unterschiedlicher Rechtsgüter breiter anzusetzen. Das zeigt das dritte Beispiel. Es geht nicht nur um Religionsfreiheit der öffentlichrechtlichen Mitarbeiter, sondern auch um das Neutralitätsgebot der öffentlichen Hand. Bekannt geworden ist in diesem Zusammenhang das Ludin-Urteil von 2003. Das Land Baden-Württemberg hatte sich trotz fachlicher Qualifikation 
geweigert, Fereshta Ludin als Beamtin auf Probe in den Schuldienst zu übernehmen, denn sie weigerte sich, religionsneutral aufzutreten. Gegen diese Entscheidung klagte Ludin vor dem Verwaltungsgericht Stuttgart, dem Verwaltungsgerichtshof Baden-Württemberg, dem Bundesverwaltungsgericht und schließlich vor dem Bundesverfassungsgericht. Das Gericht nahm nicht explizit zu der Frage Stellung, ob das Kopftuch im Schuldienst zulässig sei, sondern sah den Landesgesetzgeber, der im deutschen Föderalismus für die Normgebung in dieser Frage zuständig ist, als geeignete Instanz an, um dieses Spannungsverhältnis zu klären. Eine Mehrheit der Bundesländer entschied in der Folge, dass es Lehrkräften an staatlichen Schulen zuzumuten sei, politische, religiöse, weltanschauliche oder ähnliche äußere Bekundungen im Dienst zu unterlassen (SVR 2016).

Zwölf Jahre später ändert sich die Rechtsprechung sehr deutlich. Einer muslimischen Lehrerin und einer muslimischen Sozialpädagogin aus NordrheinWestfalen war es eben laut nordrhein-westfälischen Schulgesetzen untersagt, im Dienst ein Kopftuch (bzw. eine Mütze als Kopftuchersatz) zu tragen. Die Lehrerin bestand jedoch darauf und klagte. Der Konflikt wurde bis zum Bundesverfassungsgericht getragen, der in seinem Urteil keine generelle Gestattung religiös motivierter Bekleidungsformen im öffentlichen Dienst vorsieht, aber auch kein generelles Verbot. Im Urteil wird ausgeführt, dass jene Bekleidung zu wählen sei, die auf substantielle oder auch nur potentielle Konfliktlagen in der Schule Rücksicht nimmt. Wenn Unfrieden droht oder eine kopftuchtragende Lehrerin das Neutralitätsgebot verletzt, dann ist das Tragen eines Kopftuches nicht erlaubt, sonst schon - ein unbefriedigendes Urteil, weil es Unklarheit produziert, die Entscheidung auf die Schulstandorte und die dort Verantwortlichen abwälzt und generell zeigt, wie schwer sich Gerichte in der Abwägung von individueller Glaubensfreiheit und kollektivem Neutralitätsgebot tun.

Der letzte an dieser Stelle erwähnte Fall betrifft die bekannte, aber eigentlich recht seltene Abmeldung der Töchter muslimischer Eltern aus religiösen Gründen vom gemischtgeschlechtlichen Sport- bzw. Schwimmunterricht. Sie berufen sich dabei auf die Bekleidungsvorschriften des Korans, die Religionsfreiheit und das Erziehungsrecht der Eltern. Im September 2013 entschied in einem konkreten Fall das deutsche Bundesverwaltungsgericht - anders als noch ein Jahrzehnt zuvor -, dass es einer 11-jährigen muslimischen Schülerin zuzumuten sei, wenigstens im Burkini - einem Ganzkörperschwimmanzug - am koedukativen Schwimmunterricht teilzunehmen. Es begründete diese Entscheidung damit, dass das Grundrecht der Glaubensfreiheit grundsätzlich keinen Anspruch darauf vermittelt, im Rahmen der Schule nicht mit Verhaltensgewohnheiten Dritter konfrontiert zu werden, die außerhalb der Schule und außerhalb der religiösen Gemeinschaft verbreitet sind. Das Gericht entschied sich dagegen, die Schule als eine Art „Biotop“ zu definieren, in dem Kinder von den „Zumutungen des Alltags" (hier konkret: vom Anblick gleichaltriger Schüler in Badebekleidung) verschont bleiben sollten (BVerwG 6 C 8/91, Rn. 23; SVR 2016). Auf der Kon- 
fliktstrecke bleibt dennoch das 11-jährige Mädchen, welches zwischen den vermeintlich religiös motivierten Erziehungsansprüchen der Eltern und der sozialen Welt der Gleichaltrigen hin- und hergerissen wird.

\section{Ein Appell als Ausblick}

Religion und Religiosität besitzen für Menschen mit und ohne Migrationshintergrund unterschiedliche Bedeutungen und zwar in einem Ausmaß, welches von ihnen selbst bestimmt wird. Das gehört auch zum Kern einer freiheitlichen und säkularen Gesellschaft. Religionen versuchen, auch auf Letztfragen menschlicher Existenz Antworten zu geben, und helfen Menschen damit bei der Bewältigung der Ungewissheit von Gegenwart und Zukunft (Kontingenztheorie). Religionen haben insbesondere auch bei Integrationsprozessen eine besondere Bedeutung, weil gerade für Zugewanderte mit einer noch kurzen Aufenthaltsdauer das Bedürfnis nach Orientierung und Halt besonders wichtig ist. Religionen sollten Menschen aber bei der Bewältigung des Alltags nicht in Gewissenskonflikte drängen. Es sollte möglich sein, dass ein muslimischer Lagerarbeiter eine Bierkiste einräumt, ohne in Gewissensnöte zu geraten, ein muslimisches Mädchen am Schwimmunterricht auch im normalen Badeanzug teilnehmen kann und eine Lehrerin weltanschaulich neutral den Schülerinnen und Schülern gegenübertritt.

Gläubige und Nichtgläubige, Christen, Muslime, Juden und alle anderen Anhänger von Glaubensgemeinschaften müssen in einer pluralistischen und säkularen Gesellschaft zueinander finden und ihren Glauben in toleranter Weise praktizieren, sonst werden sie in fragwürdige Konflikte hineingezogen, bei denen keiner gewinnt, aber alle verlieren. Die Religionsgemeinschaften tragen dabei eine besondere Verantwortung, denn sie deuten und interpretieren jene Glaubensinhalte, die es den Gläubigen ermöglichen sollten, sich in einer religiös pluralen und säkularen Gesellschaft zurecht $\mathrm{zu}$ finden, ohne immer wieder in Glaubenskonflikte zu geraten. Eine gewisse Pragmatik ist dabei einzufordern und sollte immer auch möglich sein, sofern der Mensch und sein Wohlergehen im Mittelpunkt stehen sollten. Gelingt dies, dann sind die Religion und religiöse Freiheiten nicht mehr primär mit Konfliktpotenzial und Integrationshemmnissen verbunden, sondern können zum Ermöglicher von Integration werden.

\section{Literaturverzeichnis}

Bankston, Carl / Zhou, Min: Growing up American. How Vietnamese Children Adapt to Life in the United States. New York 1998.

Bommes, Michael: Erarbeitung eines operationalen Konzepts zur Einschätzung von Integrationsprozessen und Integrationsmaßnahmen. Gutachten für den Sachverständigenrat für Migration und Integration. Osnabrück 2004. 
Darnell, Alfred / Sherkat, Darren: „The Impact of Protestant Fundamentalism on Educational Attainment", in: American Sociological Review (62/2) 1997, S. 306-315.

Esser, Hartmut: Integration und ethnische Schichtung (= Arbeitspapiere des Mannheimer Zentrum für Europäische Sozialforschung 40). Mannheim 2001.

Foner, Nancy / Alba, Richard: „Immigrant Religion in the U.S. and Western Europe: Bridge or Barrier to Inclusion?“, in: International Migration Review (42/2) 2008, S. 360-392.

Glanville, Jennifer / Sikkink, David / Hernández, Edwin: „Religious Involvement and Educational Outcomes: The Role of Social Capital and Extracurricular Participation“, in: The Sociological Quarterly (49/1) 2008, S. 105-138.

Haug, Sonja / Müssig, Stephanie / Stichs, Anja: Muslimisches Leben in Deutschland. Im Auftrag der Deutschen Islam Konferenz. Forschungsbericht 6. Nürnberg 2009.

Helbig, Marcel / Schneider, Thorsten: Auf der Suche nach dem katholischen Arbeitermädchen vom Lande. Religion und Bildungserfolg im regionalen, historischen und internationalen Vergleich. Wiesbaden 2014.

Hirschman, Charles: ,The Role of Religion in the Origins and Adaptation of Immigrant Groups in the United States“, in: International Migration Review (38/3) 2004, S. 12061233.

Lehmann, Hartmut: Säkularisierung. Der europäische Sonderweg in Sachen Religion. Göttingen 2004.

Park, Robert / Burgess, Ernest: Introduction to the Science of Sociology. Chicago 1921.

Pollack, Detlef / Müller, Olaf: Religionsmonitor verstehen was verbindet Religiosität und Zusammenhalt in Deutschland. Gütersloh 2013.

Regnerus, Mark D.: „Shaping School Success: Religious Socialization and Educational Outcomes in Urban Public Schools“, in: Journal for the Scientific Study of Religion (39/3) 2000, S. 363-370.

Sander, William: „Religious Background and Educational Attainment: The Effects of Buddhism, Islam, and Judaism“, in: Economics of education review (29/3) 2010, S. 489-494.

Stichs, Anja / Müssig, Stephanie: „Muslime in Deutschland und die Rolle der Religion für die Arbeitsmarktintegration“, in: Halm, Dirk / Meyer, Hendrik (Hg.): Islam und die deutsche Gesellschaft. Wiesbaden 2013, S. 49-85.

Treibel-Illian, Anette: Migration in modernen Gesellschaften: Soziale Folgen von Einwanderung, Gastarbeit und Flucht. Mannheim / München 2011.

Winckelmann, Johannes: Max Weber, die protestantische Ethik I. Eine Aufsatzsammlung. Gütersloh 1984.

Zhou, Min: „Segmented Assimilation: Issues, Controversies and Recent Research on the New Second Generation“, in: International Migration Review (31/4) 1997, S. 9751008.

\section{Internetquellen}

Langenfeld, Christine: Religiöse Freiheit - Gefahr oder Hilfe für die Integration? Bitburger Gespräche, 2010, verfügbar unter: www.uni-trier.de/fileadmin/fb5/inst/IRP/ 
BG_Einzeldokumente_ab_2010/Bitburger_Gespr_2010_I_Langenfeld_83-98_ge schuetzt.pdf [14.02.2016].

Statistik Austria, verfügbar unter: http://www.statistik.at/web_de/statistiken/index.html [17.10.2016].

SVR (Sachverständigenrat deutscher Stiftungen für Migration und Integration): Viele Götter, ein Staat: Religiöse Vielfalt und Teilhabe im Einwanderungsland. Jahresgutachten 2016 mit Integrationsbarometer. Berlin 2016, verfügbar unter: http://www. svr-migration.de/wp-content/uploads/2016/04/SVR_JG_2016-mit-Integrationsbaro meter_WEB.pdf [17.10.2016]. 